Bull. Korean Math. Soc. 50 (2013), No. 6, pp. 1905-1914

http://dx.doi.org/10.4134/BKMS.2013.50.6.1905

\title{
STRONG MORI MODULES OVER AN INTEGRAL DOMAIN
}

\author{
Gyu Whan Chang
}

\begin{abstract}
Let $D$ be an integral domain with quotient field $K, M$ a torsion-free $D$-module, $X$ an indeterminate, and $N_{v}=\left\{f \in D[X] \mid c(f)_{v}\right.$ $=D\}$. Let $q(M)=M \otimes_{D} K$ and $M_{w_{D}}=\{x \in q(M) \mid x J \subseteq M$ for a nonzero finitely generated ideal $J$ of $D$ with $\left.J_{v}=D\right\}$. In this paper, we show that $M_{w_{D}}=M[X]_{N_{v}} \cap q(M)$ and $(M[X])_{w_{D[X]}} \cap q(M)[X]=$ $M_{w_{D}}[X]=M[X]_{N_{v}} \cap q(M)[X]$. Using these results, we prove that $M$ is a strong Mori $D$-module if and only if $M[X]$ is a strong Mori $D[X]$ module if and only if $M[X]_{N_{v}}$ is a Noetherian $D[X]_{N_{v}}$-module. This is a generalization of the fact that $D$ is a strong Mori domain if and only if $D[X]$ is a strong Mori domain if and only if $D[X]_{N_{v}}$ is a Noetherian domain.
\end{abstract}

\section{Introduction}

Let $R$ be a commutative ring with identity. For any $R$-module $A$, let

$$
A[X]=\left\{m_{0}+m_{1} X+\cdots+m_{k} X^{k} \mid m_{i} \in A\right\}
$$

be the set of all polynomials in $X$ with coefficients in $A$. Then $A[X]=A \otimes_{R}$ $R[X]$. For all $f=m_{0}+m_{1} X+\cdots+m_{k} X^{k}$ and $g=n_{0}+n_{1} X+\cdots+n_{l} X^{l}$ in $A[X]$ with $k \leq l$ and $h=a_{0}+a_{1} X+\cdots+a_{n} X^{n}$ in $R[X]$, define the addition of $f, g$ and the scalar product of $f$ and $h$ by the obvious way:

$$
\begin{aligned}
f+g= & \left(m_{0}+n_{0}\right)+\left(m_{1}+n_{1}\right) X+\cdots+\left(m_{k}+n_{k}\right) X^{k} \\
& +n_{k+1} X^{k+1}+\cdots+n_{l} X^{l}, \\
g f= & \sum_{i=1}^{k+n} c_{i} X^{i}, \text { where } c_{i}=\sum_{j+s=i} a_{j} m_{s} .
\end{aligned}
$$

It is routine to check that $A[X]$ is an $R[X]$-module [2, Exercise 6, page 32]. Let $f=m_{0}+m_{1} X+\cdots+m_{k} X^{k} \in A[X]$. As in the case of polynomial rings, we define " $f=0 \Leftrightarrow m_{0}=m_{1}=\cdots=m_{k}=0$ ", and we also say that if $m_{k} \neq 0$, then $m_{k}$ is the leading coefficient of $f$ and $k$ is the degree of $f$ denoted by $\operatorname{deg}(f)$ (For convenience, we let $\operatorname{deg}(0)=-\infty$ ). The content of a

Received May 6, 2012; Revised May 4, 2013.

2010 Mathematics Subject Classification. 13A15.

Key words and phrases. polynomial module, Noetherian module, strong Mori module. 
polynomial $f \in A[X]$, denoted by $c(f)$, is the $R$-submodule of $A$ generated by the coefficients of $f$, i.e., $c(f)=\sum_{i=0}^{k} R m_{i}$.

Let $S_{1}$ be a (saturated) multiplicative subset of $R$ with $0 \notin S_{1}$. The localization $A_{S_{1}}$ of $A$ with respect to $S_{1}$ is defined obviously, and so $A_{S_{1}}$ is an $R_{S_{1}}$-module. If $P$ is a prime ideal of $R$, we write $A_{P}$ for $A_{R \backslash P}$. We say that $A$ is an $S_{1}$-torsion-free module if $s a=0$ for $s \in S_{1}$ and $a \in A$ implies $a=0$. Clearly, if $A$ is torsion-free, then $A$ is $S$-torsion-free for any multiplicative subset $S$ of $R$ with $0 \notin S$. Note that the set $\left\{m \in A \mid s m=0\right.$ for some $\left.s \in S_{1}\right\}$ is the kernel of the canonical $R$-module homomorphism $\alpha: A \rightarrow A_{S_{1}}$ given by $m \mapsto \frac{m}{1}$. So if $A$ is an $S_{1}$-torsion-free module, then $\alpha$ is injective, and hence $A$ can be considered as an $R$-submodule of $A_{S_{1}}$; in this case, we write $m=s \cdot \frac{m}{s} \in A$ and " $\frac{m}{s}=\frac{m^{\prime}}{s^{\prime}} \Leftrightarrow s m^{\prime}=s^{\prime} m$ " for any $s, s^{\prime} \in S_{1}$ and $m, m^{\prime} \in A$.

Let $D$ be an integral domain with quotient field $K$. For any nonzero fractional ideal $I$ of $D$, define $I_{v}=\left(I^{-1}\right)^{-1}$, where $I^{-1}=\{x \in K \mid x I \subseteq D\}$, and let $I_{t}=\cup\left\{J_{v} \mid J \subseteq I\right.$ is a nonzero finitely generated ideal of $\left.D\right\}$ and $I_{w}=\{x \in K \mid x J \subseteq I$ for a nonzero finitely generated ideal $J$ of $D$ with $\left.J^{-1}=D\right\}$. Let $*=v, t$ or $w$. We say that $I$ is a $*$-ideal if $I=I_{*}$. Clearly, $I_{w} \subseteq I_{t} \subseteq I_{v}$, and hence $v$-ideals are $t$-ideals and $t$-ideals are $w$-ideals. Let $*$-Max $(D)$ denote the set of $*$-ideals maximal among proper integral $*$-ideals of $D$. It is known that $w-\operatorname{Max}(D)=t-\operatorname{Max}(D)$ and $I_{w}=\cap_{P \in t-\operatorname{Max}(D)} I D_{P}[1$, Corollary 2.13]. Also, $t-\operatorname{Max}(D) \neq \emptyset$ if $D$ is not a field. For any undefined concepts and notations, see [2], [4] or [9].

Let $M$ be a torsion-free $D$-module and $q(M)=M \otimes_{D} K$, which is the injective envelope of $M$. Let $G V(D)$ be the set of nonzero finitely generated ideals $J$ of $D$ with $J_{v}=D$. As in [10, Definition 3], we define $M_{w_{D}}=\{x \in$ $q(M) \mid J x \subseteq M$ for some $J \in G V(D)\}$ (If there is no confusion, we simply denote $w_{D}$ by $\left.w\right)$. Then $M_{w}$ is a $D$-submodule of $q(M)$ and $\left(M_{w}\right)_{w}=M_{w}[10$, Section 2]. We say that $M$ is a $w_{D}$-module (simply, $w$-module) if $M=M_{w}$; so $M_{w}$ is a $w$-module. A $w$-module $M$ is called a strong Mori module if $M$ satisfies the ascending chain condition on $w$-submodules of $M$, while $D$ is a strong Mori domain if $D$ is a strong Mori module. Clearly, Noetherian modules are strong Mori modules. Conversely, if each maximal ideal of $D$ is a $t$-ideal (e.g., onedimensional integral domain), then $N_{w}=N$ for all $D$-submodules $N$ of $M$, and thus a strong Mori module is a Noetherian module. Also, $M$ is a strong Mori $D$-module if and only if every $w$-submodule $N$ of $M$ is of finite type, i.e., $N=\left(\sum_{i=1}^{k} D m_{i}\right)_{w}$ for some $m_{1}, \ldots, m_{k} \in N$ [10, Theorem 4.4]. It is known that $A$ is a Noetherian $R$-module if and only if $A[X]$ is a Noetherian $R[X]$ module [2, Exercise 10, page 85]. Also, if we set $S=\{f \in R[X] \mid c(f)=R\}$, then $S$ is a regular multiplicative subset of $R[X]$ [4, Proposition 33.1]. So if $R$ is a Noetherian ring, then $R[X]$, and thus $R[X]_{S}$ is a Noetherian ring. Conversely, if $I$ is an ideal of $R$, then $I R[X]_{S} \cap R=I$ [4, Proposition 33.1]; so $I$ is finitely generated when $I R[X]_{S}$ is finitely generated. Thus, $R$ is a Noetherian ring if 
and only if $R[X]_{S}$ is a Noetherian ring. The purpose of this paper is to extend these results to strong Mori modules.

More precisely, let $X$ be an indeterminate and $N_{v}=\left\{f \in D[X] \mid c(f)_{v}=\right.$ $D\}$. In Section 1 , we show that $A$ is a Noetherian $R$-module if and only if $A[X]_{S}$ is a Noetherian $R[X]_{S}$-module, where $S=\{f \in R[X] \mid c(f)=R\}$. Next, in Section 2, we show that $M_{w_{D}}=M[X]_{N_{v}} \cap q(M)$ and $(M[X])_{w_{D[X]}} \cap q(M)[X]=$ $M_{w_{D}}[X]=M[X]_{N_{v}} \cap q(M)[X]$. Then we use these results to prove that $M$ is a strong Mori $D$-module if and only if $M[X]$ is a strong Mori $D[X]$-module if and only if $M[X]_{N_{v}}$ is a Noetherian $D[X]_{N_{v}}$-module. As a corollary, we have that if $0 \rightarrow M^{\prime} \rightarrow M \rightarrow M^{\prime \prime} \rightarrow 0$ is an exact sequence of torsion-free $D$-modules, then $M$ is a strong Mori module if and only if $M^{\prime}$ and $M^{\prime \prime}$ are strong Mori modules. Also, we show that if $M$ is a strong Mori $D$-module, then a $D$-module homomorphism $\phi: M \rightarrow M$ is surjective if and only if $\phi$ is bijective.

\section{Hilbert basis theorem and Noetherian modules}

Throughout this section, $R$ denotes a commutative ring with identity, $A$ is an $R$-module, $X$ is an indeterminate, and $S=\{f \in R[X] \mid c(f)=R\}$.

The Hilbert basis theorem states that $R$ is a Noetherian ring if and only if $R[X]$ is a Noetherian ring. As the module analog, it is known that $A$ is a Noetherian $R$-module if and only if $A[X]$ is a Noetherian $R[X]$-module [2, Exercise 10, page 85], which implies the Hilbert basis theorem because $R$ (resp., $R[X])$ is an $R$-module (resp., $R[X]$-module). Also, $R$ is a Noetherian ring if and only if $R[X]_{S}$ is a Noetherian ring. In this section, we extend this result to Noetherian modules.

Lemma 1.1. Let $S_{1}$ be a multiplicative subset of $R$, and assume that $A$ is an $S_{1}$-torsion-free module.

(1) If $N$ is an $R_{S_{1}}$-submodule of $A_{S_{1}}$, then $N=(N \cap A)_{S_{1}}$.

(2) If $A$ is a Noetherian $R$-module, then $A_{S_{1}}$ is a Noetherian $R_{S_{1}}$-module.

Proof. (1) Clearly, $(N \cap A)_{S_{1}} \subseteq N$. For the reverse, let $x \in N \subseteq A_{S_{1}}$, and so $x=\frac{m}{s}$ for some $m \in A$ and $s \in S_{1}$. As we noted in the introduction, $A$ can be considered as an $R$-submodule of $A_{S_{1}}$; so $s x=m \in N \cap A$. Hence $x \in(N \cap A)_{S_{1}}$. Thus $N \subseteq(N \cap A)_{S_{1}}$.

(2) Let $N$ be an $R_{S_{1}}$-submodule of $A_{S_{1}}$; then $N=(N \cap A)_{S_{1}}$ by (1). Since $N \cap A$ is an $R$-submodule of $A$, there exist some $n_{1}, \ldots, n_{k} \in N \cap A$ such that $N \cap A=\sum_{i=1}^{k} R n_{i}\left[2\right.$, Proposition 6.2], and hence $N=(N \cap A)_{S_{1}}=$ $\sum_{i=1}^{k} R_{S_{1}} n_{i}$. Thus $A_{S_{1}}$ is a Noetherian $R_{S_{1}}$-module [2, Proposition 6.2].

Lemma 1.2. Let $N$ be an $R[X]$-submodule of $A[X]$ and let $N_{k}$ be the set of leading coefficients of polynomials of degree $\leq k$ in $N$. Then $N_{k}$ is an $R$ submodule of $A$.

Proof. Let $a, b \in N_{k}$, and let $f, g \in A[X] \operatorname{such}$ that $\operatorname{deg}(f) \leq \operatorname{deg}(g) \leq k$ and $a, b$ are the leading coefficients of $f, g$, respectively. Clearly, $N_{k} \subseteq A$. Since 
$A[X]$ is an $R[X]$-module, we have $h:=X^{\operatorname{deg}(g)-\operatorname{deg}(f)} f \pm g \in A[X]$ and $\operatorname{deg}(h) \leq$ $\operatorname{deg}(g) \leq k$. If $a \pm b=0$, then $a \pm b \in N_{-\infty} \subseteq N_{k}$. If $a \pm b \neq 0$, then $a \pm b$ is the leading coefficient of $h$, and hence $a \pm b \in N_{k}$. Thus $N_{k}$ is a subgroup of $A$. Next, if $r \in R$, then $r f \in A[X]$. If $r a=0$, then $r a \in N_{k}$. If $r a \neq 0$, then $r a$ is the leading coefficient of $r f$ and $\operatorname{deg}(r f)=\operatorname{deg}(f) \leq k$; so $r a \in N_{k}$. Thus $N_{k}$ is an $R$-submodule of $A$.

The Dedekind-Mertens lemma states that if $f, g \in R[X]$ with $\operatorname{deg}(g)=m$, then $c(f)^{m+1} c(g)=c(f)^{m} c(f g)$ (see, for example, [4, Theorem 28.1]). The next result is the module analog, whose proof is the same as that of $[4$, Theorem 28.1].

Proposition 1.3 ([9, Theorem 1.8.11]). If $f \in R[X]$ and $g \in A[X]$, then $c(f)^{m+1} c(g)=c(f)^{m} c(f g)$ for some integer $m \geq 1$.

We next give the main result of this section. For easy reference of the reader, we also give the proof of the fact that $A$ is a Noetherian $R$-module if and only if $A[X]$ is a Noetherian $R[X]$-module.

Theorem 1.4. For any $R$-module $A$, the following statements are equivalent.

(1) $A$ is a Noetherian $R$-module.

(2) $A[X]$ is a Noetherian $R[X]$-module.

(3) $A[X]_{S}$ is a Noetherian $R[X]_{S}$-module, where $S=\{f \in R[X] \mid c(f)$ $=R\}$.

Proof. $(1) \Rightarrow(2)$ It suffices to show that every submodule of $A[X]$ is finitely generated. Let $B$ be an $R[X]$-submodule of $A[X]$, and let $N_{k}$ be the set of leading coefficients of polynomials in $B$ of degree $\leq k$. Then $N_{k}$ is an $R$ submodule of $A$ by Lemma 1.2 and $N_{0} \subseteq N_{1} \subseteq N_{2} \subseteq \cdots \subseteq A$. Let $N=$ $\cup_{k \geq 1} N_{k}$. Since $A$ is a Noetherian $R$-module, all the $N_{k}$ and $N$ are finitely generated $R$-submodules of $A$. Let $f_{1}, \ldots, f_{n} \in B$ such that $f_{i}=a_{i} X^{r_{i}}+$ (lower terms) and $N=\sum_{i=1}^{n} R a_{i}$. Let $r=\max \left\{r_{1}, \ldots, r_{n}\right\}$. For each $j$ from 1 to $r-1$, pick $g_{j 1}, \ldots, g_{j k_{j}} \in B$ such that $g_{j i}=b_{j i} X^{r_{j i}}+$ (lower terms) and $N_{j}=\sum_{i=1}^{k_{j}} R b_{j i}$.

Let $f=a X^{m}+($ lower terms $) \in B$; then $a \in N$, and hence $a=u_{1} a_{1}+u_{2} a_{2}+$ $\cdots+u_{n} a_{n}$ for some $u_{i} \in R$. If $m \geq r$, then $f-\sum_{i=1}^{n} u_{i} X^{m-r_{i}} f_{i} \in B$ and has degree $\leq m-1$. Repeating this process, we have polynomials $h_{i} \in R[X]$ such that $h^{(0)}:=f-\sum_{i=1}^{n} h_{i} f_{i} \in B$ and $\operatorname{deg}\left(h^{(0)}\right)=k_{h^{(0)}} \leq r-1$. Next, note that the leading coefficient of $h^{(0)}$ is in $N_{k_{h^{(0)}}}$; so there are polynomials $h_{i}^{\prime} \in R[X]$ such that $h^{(1)}:=h^{(0)}-\sum_{i} h_{i}^{\prime} g_{k_{h^{(0)}}} \in B$ and $\operatorname{deg}\left(h^{(1)}\right) \leq k_{h^{(0)}}-1$. This process continues until $h^{(i)}=0$, because $\operatorname{deg}\left(h^{(0)}\right)>\operatorname{deg}\left(h^{(1)}\right)>\operatorname{deg}\left(h^{(2)}\right)>$ $\cdots \geq 0$ cannot contain more than $\operatorname{deg}\left(h^{(0)}\right)$ integers. Hence $f \in \sum_{i=1}^{n} R[X] f_{i}+$ $\sum_{j, i} R[X] g_{j i}$. Thus we have $B=\sum_{i=1}^{n} R[X] f_{i}+\sum_{j, i} R[X] g_{j i}$.

$(2) \Rightarrow(3)$ Let $f \in S$ and $g \in A[X]$ such that $f g=0$. Then, since $c(f)=R$, by Proposition 1.3 we have $c(g)=c(f g)=(0)$; so $g=0$. Hence $A[X]$ is $S$ torsion-free, and thus by Lemma $1.1(2), A[X]_{S}$ is a Noetherian $R[X]_{S}$-module. 
(3) $\Rightarrow(1)$ Suppose that $N$ is an $R$-submodule of $A$. Then $N[X]$ is an $R[X]$ submodule of $A[X]$, and hence $N[X]_{S}$ is an $R[X]_{S}$-submodule of $A[X]_{S}$. Let $f_{1}, \ldots, f_{k} \in N[X]$ such that $N[X]_{S}=\sum_{i=1}^{k} R[X]_{S} f_{i}$. Note that $N \subseteq N[X]_{S}$; so if $a \in N$, then there exist polynomials $h_{1}, \ldots, h_{k} \in R[X]$ and $g_{1}, \ldots, g_{k} \in S$ such that $a=\sum_{i=1}^{k} \frac{h_{i}}{g_{i}} f_{i} \in\left(\sum_{i=1}^{k} c\left(f_{i}\right)\right)[X]_{S} \subseteq N[X]_{S}$. So $a \in \sum_{i=1}^{k} c\left(f_{i}\right)$. Hence $N \subseteq \sum_{i=1}^{k} c\left(f_{i}\right)$, and thus $N=\sum_{i=1}^{k} c\left(f_{i}\right)$. Thus $N$ is finitely generated, so $A$ is a Noetherian $R$-module [2, Proposition 6.2].

\section{Strong Mori modules}

Let $D$ denote an integral domain with quotient field $K, M$ a torsion-free $D$ module, $q(M)=M \otimes_{D} K, X$ an indeterminate, $N_{v}=\left\{f \in D[X] \mid c(f)_{v}=D\right\}$.

Note that, since $M$ is torsion-free, $\frac{m}{s}=\frac{m^{\prime}}{s^{\prime}}$ if and only if $s^{\prime} m=s m^{\prime}$ for $m, m^{\prime} \in M$ and $s, s^{\prime} \in D \backslash\{0\}$. Also, if $f=m_{0}+m_{1} X+\cdots+m_{k} X^{k} \in M[X]$ with $m_{k} \neq 0$ and $h=a_{0}+a_{1} X+\cdots+a_{n} X^{n} \in D[X]$ with $a_{n} \neq 0$, then $h f=$ $a_{n} m_{k} X^{n+k}+$ (lower terms), and since $M$ is torsion-free, we have $a_{n} m_{k} \neq 0$, and hence $h f \neq 0$. Thus, $M[X]$ is a torsion-free $D[X]$-module.

Lemma 2.1. (1) $q(M)=M_{D \backslash\{0\}}$.

(2) If $S$ is a multiplicative subset of $D$, then $M_{S}$ is a $D_{S}$-module.

(3) $M$ is a D-submodule of $q(M)$.

(4) $M[X]_{N_{v}}$ is a $D[X]_{N_{v}}$-module.

(5) If $S \subseteq T$ are multiplicative subsets of $D$, then $\left(M_{S}\right)_{T}=M_{T} \subseteq q(M)$.

Proof. (1) [7, Theorem 4.4]. (2) See [7, page 25]. (3) If we let $S=D \backslash\{0\}$, then $M_{S}=q(M)$ by (1), and since $M$ is $S$-torsion-free, $M$ is a $D$-submodule of $q(M)$. (4) This is an immediate consequence of (2) because $N_{v}$ is a multiplicative subset of $D[X]$. (5) Clear.

Lemma 2.2. Let $M$ be a w-module and $P \in t$-Max(D). If $N$ is a $D_{P^{-}}$ submodule of $M_{P}$, then

(1) $N=(N \cap M)_{P}$ and

(2) $N \cap M$ is a w-module.

Proof. (1) This follows directly from Lemma 1.1(1) because $M$ is a torsion-free $D$-module.

(2) Let $x \in(N \cap M)_{w}$. Let $J \in G V(D)$ such that $J x \subseteq N \cap M$; then $J \nsubseteq P$. Choose $a \in J \backslash P$. Then $a x \in J x \subseteq N \cap M$, and hence $x=\frac{a x}{a} \in(N \cap M)_{P}=N$. So $(N \cap M)_{w} \subseteq N \cap M$, and thus $(N \cap M)_{w}=N \cap M$.

Proposition 2.3. If $M$ is a strong Mori D-module, then $M_{P}$ is a Noetherian $D_{P}$-module for all $P \in t$-Max $(D)$.

Proof. Let $N_{1} \subseteq N_{2} \subseteq \cdots$ be an ascending chain of $D_{P}$-submodules of $M_{P}$. Then $N_{1} \cap M \subseteq N_{2} \cap M \subseteq \cdots$ is an ascending chain of $w$-submodules of $M$ over $D$ by Lemma 2.2(2). So there exists a positive integer $k$ such that 
$N_{k} \cap M=N_{k+i} \cap M$ for $i=1,2,3, \ldots$ Thus by Lemma 2.2(1), we have $N_{k}=\left(N_{k} \cap M\right)_{P}=\left(N_{k+i} \cap M\right)_{P}=N_{k+i}$ for $i=1,2,3, \ldots$.

It is known that $I_{w}=I D[X]_{N_{v}} \cap K=\cap_{P \in t-\operatorname{Max}(D)} I D_{P}$ for all nonzero fractional ideals $I$ of $D$. Our next result is a torsion-free module analog.

Lemma 2.4. (1) $M_{P}=\left(M_{w_{D}}\right)_{P}$ for all $P \in t$-Max $(D)$.

(2) $M_{w_{D}}=M[X]_{N_{v}} \cap q(M)=\cap_{P \in t-\operatorname{Max}(D)} M_{P}$.

(3) $M[X]_{N_{v}}=M_{w_{D}}[X]_{N_{v}}$.

(4) $(M[X])_{w_{D[X]}} \cap q(M)[X]=M_{w_{D}}[X]$.

(5) $M_{w_{D}}[X]=M[X]_{N_{v}} \cap q(M)[X]$.

Proof. (1) [10, page 1297].

(2) It is known that $M_{w}=\cap_{P \in t-\operatorname{Max}(D)} M_{P}[1$, p. 2463]; so it suffices to show that $M_{w}=M[X]_{N_{v}} \cap q(M)$ :

$(\subseteq)$ Let $0 \neq x \in M_{w}$. Then there exists an $I \in G V(D)$ such that $x I \subseteq M$. So if we choose an $f \in D[X]$ with $c(f)=I$, then $f \in N_{v}$, and thus $x=\frac{x f}{f} \in$ $M[X]_{N_{v}} \cap q(M)$.

() Let $m \in M[X]_{N_{v}} \cap q(M)$. Then $m=\frac{g}{f}$ for some $g \in M[X]$ and $f \in N_{v}$, so $f m=g$. Hence $(c(f)) m=c(f m)=c(g) \subseteq M$, and thus $m \in M_{w}$ because $c(f) \in G V(D)$.

(3) This follows directly from (2).

(4) Put $R=D[X]$. Let $t$ be an indeterminate over $R, N=\{f \in R[t] \mid$ $\left.c(f)_{v}=R\right\}$, and $N_{v}(t)=\left\{g \in D[t] \mid c(g)_{v}=D\right\}$. Recall that $(I D[X])_{v}=$ $I_{v} D[X]$ for a nonzero ideal $I$ of $D$ [5, Proposition 4.3]; so $N_{v}(t) \subseteq N$, and hence $M_{w}=M[t]_{N_{v}(t)} \cap q(M) \subseteq(M[X])[t]_{N} \cap q(M[X])=(M[X])_{w}$ by (2). Thus $M_{w}[X] \subseteq(M[X])_{w} \cap q(M)[X]$.

Conversely, let $g \in(M[X])_{w} \cap q(M)[X]$, and let $J=\left(f_{1}, \ldots, f_{s}\right)$ be a finitely generated ideal of $D[X]$ such that $J_{v}=D[X]$ and $J g \subseteq M[X]$. Let $m$ be a positive integer such that $c\left(f_{i}\right)^{m+1} c(g)=c\left(f_{i}\right)^{m} c\left(f_{i} g\right)$ for $i=1, \ldots, s$ by Proposition 1.3. Then $\left(c\left(f_{1}\right)^{m+1}+\cdots+c\left(f_{s}\right)^{m+1}\right) c(g)=c\left(f_{1}\right)^{m} c\left(f_{1} g\right)+\cdots+$ $c\left(f_{s}\right)^{m} c\left(f_{s} g\right) \subseteq M$. Since $\left(\sum c\left(f_{i}\right)\right)_{v}=D$ [5, Lemma 4.2], $\left(c\left(f_{1}\right)^{m+1}+\cdots+\right.$ $\left.c\left(f_{s}\right)^{m+1}\right)_{v}=D$, and hence $c(g) \subseteq M_{w}$. Thus $g \in M_{w}[X]$.

(5) Since $M_{w} \subseteq M[X]_{N_{v}} \cap q(M)$ by $(2), M_{w}[X] \subseteq M[X]_{N_{v}} \cap q(M)[X]$. For the reverse containment, let $f=\frac{h}{g} \in M[X]_{N_{v}} \cap q(M)[X]$, where $g \in N_{v}$ and $h \in M[X]$. Then $f g=h$, and hence $c(g)^{m+1} c(f)=c(g)^{m} c(f g)=c(g)^{m} c(h) \subseteq$ $M$ for some $m \geq 1$ by Proposition 1.3. Note that $c(g)^{m+1}$ is finitely generated and $\left(c(g)^{m+1}\right)_{v}=\left(\left(c(g)_{v}\right)^{m+1}\right)_{v}=D$; hence $c(g)^{m+1} \in G V(D)$, and thus $c(f) \in M_{w}$ or $f \in M_{w}[X]$. Thus $M[X]_{N_{v}} \cap q(M)[X] \subseteq M_{w}[X]$.

We next give the main result of this paper, which generalizes the fact that $D$ is a strong Mori domain if and only if $D[X]$ is a strong Mori domain if and only if $D[X]_{N_{v}}$ is a Noetherian domain [3, Theorem 2.2] (Note that an integral domain $R$ is a strong Mori (resp., Noetherian) domain if and only if $R$ is a strong Mori (resp., Noetherian) $R$-module). 
Theorem 2.5. The following statements are equivalent for a $w$-module $M$.

(1) $M$ is a strong Mori D-module.

(2) $M[X]$ is a strong Mori $D[X]$-module.

(3) $M[X]_{N_{v}}$ is a Noetherian $D[X]_{N_{v}}$-module.

Proof. (1) $\Rightarrow(3)$ Suppose that $M$ is a strong Mori $D$-module, and let $B^{\prime}$ be a $D[X]_{N_{v}}$-submodule of $M[X]_{N_{v}}$. Then $B^{\prime}=B_{N_{v}}$, where $B=B^{\prime} \cap M[X]$, by Lemma 1.1 , so it suffices to show that $B_{N_{v}}$ is a finitely generated $D[X]_{N_{v}}$ module. Let $N_{k}$ be the set of the leading coefficients of polynomials in $B$ of degree $\leq k$; then $N_{k}$ is a $D$-submodule of $M$ by Lemma 1.2 and $N_{0} \subseteq N_{1} \subseteq$ $N_{2} \subseteq \cdots$. Set $N=\cup_{k \geq 0}\left(N_{k}\right)_{w}$. Since $M$ is a strong Mori module, all the $\left(N_{k}\right)_{w}$ are finite type $w$-submodules of $M$ and $N=\left(N_{s}\right)_{w}$ for some $s$.

Let $f_{1}, \ldots, f_{k} \in B$ such that $f_{i}=m_{i} X^{r_{i}}+($ lower terms $)$ and $N=\left(\sum_{i=1}^{k} D m_{i}\right)_{w}$. Let $r=\max \left\{r_{1}, \ldots, r_{k}\right\}$. For each $j$ from 1 to $r-1$, choose $g_{j 1}, \ldots, g_{j k_{j}} \in B$ such that $g_{j i}=b_{j i} X^{r_{j i}}+\left(\right.$ lower terms) and $\left(N_{j}\right)_{w}=\left(\sum_{i=1}^{k_{j}} D b_{j i}\right)_{w}$. We claim that $B_{N_{v}}=\sum_{i} D[X]_{N_{v}} f_{i}+\sum_{j, m} D[X]_{N_{v}} g_{j m}$.

Let $A=\sum_{i} D[X]_{N_{v}} f_{i}+\sum_{j, m} D[X]_{N_{v}} g_{j m}$, and let $Q$ be a maximal ideal of $D[X]_{N_{v}}$. Then $A=A_{N_{v}}$ and $Q=P[X]_{N_{v}}$ for some $P \in t-\operatorname{Max}(D)$. Note that $M_{P}$ is a Noetherian $D_{P}$-module by Proposition 2.3 ; so $M_{P}[X]$ is a Noetherian $D_{P}[X]$-module by Theorem 1.4. Also, since $N_{P}=\sum_{i=1}^{k} D_{P} m_{i}$ and $\left(N_{j}\right)_{P}=$ $\sum_{i=1}^{k_{j}} D_{P} b_{j i}$ by Lemma 2.4(1), the proof of $(1) \Rightarrow(2)$ of Theorem 1.4 shows that $B_{D \backslash P}=A_{D \backslash P}$. Thus by Lemma 2.1(5),

$$
\begin{aligned}
\left(B_{N_{v}}\right)_{Q} & =\left(B_{N_{v}}\right)_{P[X]_{N_{v}}}=B_{P[X]}=\left(B_{D \backslash P}\right)_{P D_{P}[X]} \\
& =\left(A_{D \backslash P}\right)_{P D_{P}[X]}=A_{P[X]}=\left(A_{N_{v}}\right)_{P[X]_{N_{v}}}=\left(A_{N_{v}}\right)_{Q} .
\end{aligned}
$$

Since $Q$ is an arbitrary maximal ideal of $D[X]_{N_{v}}$, we conclude $B_{N_{v}}=A_{N_{v}}=$ A.

$(3) \Rightarrow(2)$ Let $t$ be an indeterminate over $D[X], \mathcal{M}=M[t]$, and $R=D[t]$. By replacing $t$ with $X$, it suffices to show that $\mathcal{M}$ is a strong Mori $R$-module. Note that $\left(M[X]_{N_{v}}\right)[t]=\mathcal{M}[X]_{N_{v}}$ and $\left(D[X]_{N_{v}}\right)[t]=R[X]_{N_{v}} ;$ so $\mathcal{M}[X]_{N_{v}}$ is a Noetherian $R[X]_{N_{v}}$-module by (3) and Theorem 1.4. Let $N=\{g \in$ $\left.R[X] \mid c(g)_{v}=R\right\}$; then $N_{v} \subseteq N$ (see the proof of Lemma 2.4(4)), and hence $\left(\mathcal{M}[X]_{N_{v}}\right)_{N}=\mathcal{M}[X]_{N}$ and $\left(R[X]_{N_{v}}\right)_{N}=R[X]_{N}$ by Lemma 2.1(5). Hence, by Theorem 1.4, $\mathcal{M}[X]_{N}$ is a Noetherian $R[X]_{N}$-module.

Let $M_{1} \subseteq M_{2} \subseteq \cdots$ be an ascending chain of $w$-submodules of $\mathcal{M}$ over $R$. Then $M_{1}[X]_{N} \subseteq M_{2}[X]_{N} \subseteq \cdots$ is an ascending chain of $R[X]_{N}$-submodules of $\mathcal{M}[X]_{N}$. So there exists a positive integer $k$ such that $M_{k}[X]_{N}=M_{k+i}[X]_{N}$ for $i=1,2,3, \ldots$ Thus by Lemma $2.4(2)$, we have $M_{k}=M_{k}[X]_{N} \cap q(\mathcal{M})=$ $M_{k+i}[X]_{N} \cap q(\mathcal{M})=M_{k+i}$ for $i=1,2,3, \ldots$ Thus $\mathcal{M}$ is a strong Mori $R$ module.

(2) $\Rightarrow(1)$ Let $M_{1} \subseteq M_{2} \subseteq \cdots$ be an ascending chain of $w$-submodules of $M$. Then $\left(M_{1}[X]\right)_{w} \subseteq\left(M_{2}[X]\right)_{w} \subseteq \cdots$ is an ascending chain of $w$-submodules of $(M[X])_{w}$ over $D[X]$. So there exists a positive integer $k$ such that $\left(M_{k}[X]\right)_{w}=$ 
$\left(M_{k+i}[X]\right)_{w}$ for $i=1,2,3, \ldots$ Thus by Lemma $2.4(4)$, we have

$$
M_{k}=\left(M_{k}[X]\right)_{w} \cap q(M)=\left(M_{k+i}[X]\right)_{w} \cap q(M)=M_{k+i} \text { for } i=1,2,3, \ldots
$$

Corollary 2.6 ([10, Theorem 4.5]). D is an SM domain if and only if every finite type $w$-module $M$ over $D$ is a strong Mori module.

Proof. Suppose that $D$ is an SM domain, and let $M=N_{w}$ for some finitely generated $D$-submodule $N$ of $M$; so $M[X]_{N_{v}}=N[X]_{N_{v}}$ by Lemma 2.4(3). Hence $M[X]_{N_{v}}$ is a finitely generated $D[X]_{N_{v}}$-module, and since $D[X]_{N_{v}}$ is Noetheian [3, Theorem 2.2], $M[X]_{N_{v}}$ is a Noetherian $D[X]_{N_{v}}$-module [2, Proposition 6.5]. Thus $M$ is a strong Mori module by Theorem 2.5. The converse follows because $D$ is a finite type $w$-module over $D$ itself.

Let $R$ be a commutative ring with identity. It is well known that if $M_{1}, \ldots$, $M_{k}$ are Noetherian $R$-modules, then $\bigoplus_{i=1}^{k} M_{i}$ is also a Noetherian $R$-module. This follows directly from the fact that if $0 \rightarrow L \rightarrow M \rightarrow N \rightarrow 0$ is an exact sequence of $R$-modules, then $M$ is Noetherian if and only if $L$ and $N$ are Noetherian [2, Proposition 6.3]. We next generalize this result to strong Mori module, which shows that if $D$ is a strong Mori domain, then $D^{n}=\bigoplus_{i=1}^{n} D_{i}$, where $D_{i}=D$, is a strong Mori $D$-module for any positive integer $n$ (see Corollary 2.8).

Corollary 2.7 (cf. [8, Proposition 3.5(2)]). Let $0 \rightarrow M^{\prime} \stackrel{\alpha}{\longrightarrow} M \stackrel{\beta}{\longrightarrow} M^{\prime \prime} \rightarrow 0$ be an exact sequence of torsion-free D-modules. If $M^{\prime}, M$, and $M^{\prime \prime}$ are $w$ modules, then $M$ is a strong Mori module if and only if $M^{\prime}$ and $M^{\prime \prime}$ are strong Mori modules.

Proof. For each $f=m_{0}+m_{1} X+\cdots+m_{k} X^{k} \in M^{\prime}[X]$, define

$$
\alpha^{\prime}(f)=\sum_{i=0}^{k} \alpha\left(m_{i}\right) X^{i} .
$$

It is obvious that the map $\alpha^{\prime}: M^{\prime}[X] \rightarrow M[X]$, given by $f \mapsto \alpha^{\prime}(f)$, is an $D[X]$-module homomorphism. Also, the map $\beta^{\prime}: M[X] \rightarrow M^{\prime \prime}[X]$, defined by $\beta^{\prime}\left(\sum_{i=0}^{k} m_{i} X^{i}\right)=\sum_{i=0}^{k} \beta\left(m_{i}\right) X^{i}$, is a $D[X]$-module homomorphism. Moreover, since the sequence $0 \rightarrow M^{\prime} \stackrel{\alpha}{\longrightarrow} M \stackrel{\beta}{\longrightarrow} M^{\prime \prime} \rightarrow 0$ is sequence, it follows that $0 \rightarrow M^{\prime}[X] \stackrel{\alpha^{\prime}}{\longrightarrow} M[X] \stackrel{\beta^{\prime}}{\longrightarrow} M^{\prime \prime}[X] \rightarrow 0$ is an exact sequence of $D[X]$ modules; hence $0 \rightarrow M^{\prime}[X]_{N_{v}} \stackrel{\left(N_{v}\right)^{-1} \alpha^{\prime}}{\longrightarrow} M[X]_{N_{v}} \stackrel{\left(N_{v}\right)^{-1} \beta^{\prime}}{\longrightarrow} M^{\prime \prime}[X]_{N_{v}} \rightarrow 0$ is an exact sequence [2, Proposition 3.3]. Thus by Theorem 2.5 and [2, Proposition $6.3]$, we have that $M$ is a strong Mori module $\Leftrightarrow M[X]_{N_{v}}$ is a Noetherian module $\Leftrightarrow M^{\prime}[X]_{N_{v}}$ and $M^{\prime \prime}[X]_{N_{v}}$ are Noetherian $\Leftrightarrow M^{\prime}$ and $M^{\prime \prime}$ are strong Mori modules.

Corollary 2.8 ([8, Corollary 3.2] or [11, Proposition 4.5]). If $M_{i}(i=1, \ldots, n)$ are strong Mori D-modules, then $\bigoplus_{i=1}^{n} M_{i}$ is also a strong Mori D-module. 
Proof. This can be proved by induction on $n$ and Corollary 2.7 applying to the exact sequence $0 \rightarrow M_{n} \longrightarrow \bigoplus_{i=1}^{n} M_{i} \longrightarrow \bigoplus_{i=1}^{n-1} M_{i} \rightarrow 0$.

The following lemma is a variant of [6, Corollary 2.15].

Lemma 2.9. Let $M$ be a $w$-module. If $\varphi: M \rightarrow M$ is a module homomorphism, then the kernel of $\varphi$ is a $w$-module.

Proof. Let $N$ be the kernel of $\varphi$. If $x \in N_{w}$, there exists a $J \in G V(D)$ such that $J x \in N$. Choose $0 \neq a \in J$; then $a x \in N$, and hence $0=\varphi(a x)=a \varphi(x)$. Since $M$ is torsion-free, we have $\varphi(x)=0$, and hence $x \in N$. Thus $N_{w} \subseteq N$.

Theorem 2.10. Let $M$ be a strong Mori D-module and $\varphi: M \rightarrow M$ be a $D$-module homomorphism. If $\varphi$ is surjective, then $\varphi$ is an isomorphism.

Proof. It suffices to prove that $\varphi$ is injective. Let $\varphi^{2}=\varphi \circ \varphi$ and $\varphi^{n}=\varphi^{n-1} \circ \varphi$ for all integers $n \geq 2$. Clearly, $\varphi^{n}$ is a $D$-module homomorphism from $M$ onto itself. Hence $\operatorname{ker}\left(\varphi^{n}\right)$, the kernel of $\varphi^{n}$, is a $w$-module by Lemma 2.9, and since $\operatorname{ker}(\varphi) \subseteq \operatorname{ker}\left(\varphi^{2}\right) \subseteq \cdots$, there exists a positive integer $k$ such that $\operatorname{ker}\left(\varphi^{k}\right)=\operatorname{ker}\left(\varphi^{k+i}\right)$ for $i=1,2, \ldots$ Let $u \in \operatorname{ker}(\varphi)$. Then $u \in \operatorname{ker}\left(\varphi^{k}\right)$. Since $\varphi^{k}$ is onto, there exists $v \in M$ such that $u=\varphi^{k}(v)$. Then $0=\varphi^{k}(u)=\varphi^{2 k}(v)$; so $v \in \operatorname{ker}\left(\varphi^{2 k}\right)=\operatorname{ker}\left(\varphi^{k}\right)$, and hence $u=\varphi^{k}(v)=0$. Thus $\varphi$ is injective.

Remark 2.11. As in [8], we say that $M$ is of finite type if there is a finitely generated submodule $B$ of $M$ such that $M_{P}=B_{P}$ for all $P \in w-\operatorname{Max}(D)$; $M$ is $w$-Noetherian if every submodule of $M$ is of finite type; and a sequence $A \rightarrow B \rightarrow C$ of modules is $w$-exact if the sequence $A_{P} \rightarrow B_{P} \rightarrow C_{P}$ is exact for any maximal $w$-ideal $P$ of $D$. Wang proved that if $0 \rightarrow A \rightarrow B \rightarrow C \rightarrow 0$ is $w$-exact, then $B$ is $w$-Noetherian if and only if $A$ and $C$ are $w$-Noetherian [8, Proposition 3.5(2)] and that $\bigoplus_{i=1}^{n} M_{i}$ is $w$-Noetherian if and only if each $M_{i}$ is $w$-Noetherian [8, Corollary 3.2] (The definitions and results of [8] are given in a more general setting of commutative rings with zero divisors). By Lemma 2.4(1) and (2), if $M$ is a $w$-module, then $M$ is $w$-Noetherian if and only if $M$ is a strong Mori module. Thus, Corollaries 2.7 and 2.8 follow directly from [8, Proposition 3.5(2) and Corollary 3.2].

Acknowledgements. The author would like to thank the referees for their several valuable comments and suggestions (including an information on the paper [8]). This work was supported by the University of Incheon Research Fund in 2012 (2012-0045).

\section{References}

[1] D. D. Anderson and S. J. Cook, Two star-operations and their induced lattices, Comm. Algebra 28 (2000), no. 5, 2461-2475.

[2] M. F. Atiyah and I. G. MacDonald, Introduction to Commutative Algebra, AddisonWesley, 1969.

[3] G. W. Chang, Strong Mori domains and the ring $D[X]_{N_{v}}$, J. Pure Appl. Algebra 197 (2005), no. 1-3, 293-304. 
[4] R. Gilmer, Multiplicative Ideal Theory, Marcel Dekker, New York, 1972.

[5] J. R. Hedstrom and E. G. Houston, Some remarks on star-operations, J. Pure Appl. Algebra 18 (1980), no. 1, 37-44.

[6] H. Kim, Module-theoretic characterizations of t-linkative domains, Comm. Algebra $\mathbf{3 6}$ (2008), no. 5, 1649-1670.

[7] H. Matsumura, Commutative Ring Theory, Cambridge Univ. Press, 1986.

[8] F. Wang, Finitely presented type modules and w-coherent rings, J. Sichuan Normal Univ. 33 (2010), 1-9.

[9] _ Foundations of Commutative Ring Theory, unpublished book.

[10] F. Wang and R. L. McCasland, On w-modules over strong Mori domains, Comm. Algebra 25 (1997), no. 4, 1285-1306.

[11] H. Yin, F. Wang, X. Zhu, and Y. Chen, w-modules over commutative rings, J. Korean Math. Soc. 48 (2011), no. 1, 207-222.

Department of Mathematics

INCHEON NATIONAL UNIVERSITY

INCHEON 406-772, KoREA

E-mail address: whan@incheon.ac.kr 